\title{
Distribution pattern of tumor associated macrophages predicts the prognosis of gastric cancer
}

\author{
Jiu-Yang Liu ${ }^{1}$, Chun-wei Peng1, Gui-Fang Yang ${ }^{2}$, Wen-Qing Hu ${ }^{3}$, Xiao-Jun Yang ${ }^{1}$, \\ Chao-Qun Huang ${ }^{1}$, Bin Xiong ${ }^{1}$ and Yan $\mathrm{Li}^{1,4}$ \\ ${ }^{1}$ Department of Gastrointestinal Surgery, Zhongnan Hospital of Wuhan University, Hubei Key Laboratory of Tumor Biological \\ Behaviors \& Hubei Cancer Clinical Study Center, Wuhan, China \\ ${ }^{2}$ Department of Pathology, Zhongnan Hospital of Wuhan University, Wuhan, China \\ ${ }^{3}$ Department of Surgery, The First Affiliated Hospital of Shanxi Medical University, Taiyuan, 030001, PR China \\ ${ }^{4}$ Department of Peritoneal Cancer Surgery, Cancer Center of Beijing Shijitan Hospital Affiliated to the Capital Medical \\ University, Beijing, China \\ Correspondence to: Chun-wei Peng, email: whupengcw@whu.edu.cn \\ Yan Li, email: liyansd2@163.com \\ Keywords: gastric cancer, tumor microenvironment, macrophages, prognosis \\ Received: June 15,2017Ａccepted: September 08, $2017 \quad$ Published: October 06, 2017 \\ Copyright: Liu et al. This is an open-access article distributed under the terms of the Creative Commons Attribution License 3.0 \\ (CC BY 3.0), which permits unrestricted use, distribution, and reproduction in any medium, provided the original author and source \\ are credited.
}

\section{ABSTRACT}

Purpose: As mayor biomarkers in tumor microenvironment (TME), tumor associated macrophages (TAMs) of gastric cancer (GC) still needs further studies in terms of the number and distribution pattern.

Methods: Herein, tissue microarrays (TMA) incorporating 494 GC surgical samples in duplicate were stained for TAMs infiltration analysis. TAMs number was counted according to the locations, including infiltrating macrophages in cancer nest (MC), in invasive front (MF) and in stroma (MS). Correlations between TAMs number, distribution pattern and clinic-pathological features and survival analyses were performed.

Results: Infiltrating macrophages number in GC tissues was much higher than that in peritumoral tissues. TAMs number was not significantly correlated with the overall survival (OS). TAMs distribution pattern could be categorized into MC or MF/MS dominant pattern, and correlated with histological grade $(P=0.001)$. The median OS of MF/MS dominant pattern (22.1, 95\%CI: 23.5-28.9) was significantly shorter than that of MC dominant pattern $(25.6,95 \% \mathrm{CI}: 28.5-35.6)(P=0.002)$. By receiver operating characteristic curve (ROC) analysis, the predictive value of TAMs distribution pattern was superior to histological grade and pM stage, but inferior to pN and TNM stage.

Conclusions: TAMs distribution pattern could be an independent prognostic factor for the OS of GC patients, and patients with MF/MS dominant pattern had worse outcomes.

\section{INTRODUCTION}

Tumor microenvironment (TME) plays an important role in cancer progression and metastasis $[1,2]$. Within TME, distinct immune cells are recruited by cancerderived signals and mutually interact with cancer cells [3]. Tumor associated macrophages (TAMs), the most abundant immune-related stromal cells [4], act as key orchestrators in TME, by directly attacking cancer cells, or promoting cancer progression by suppressing antitumor immunity, or inducing angiogenesis [5].

Significant advances have been made in TAMs studies regarding their impacts on clinical outcomes. The clinical significance of TAMs can be influenced by the 
number, phenotypes and distributions at each pathological stage [6]. Studies in gastric cancer (GC) have shown that higher number of TAMs is associated with worse prognosis $[7,8]$. However, other reports have found that a higher level of TAMs infiltration results in a better outcome [9]. Therefore, it becomes controversial that TAMs emerge as significant but opposite predictors of survival for GC $[10,11]$. These conflicting results could be due to the fact that most studies pay attention to the ratios of TAMs with different phenotypic features $[12,13]$, or ignorance of TAMs distributions by simply focusing on the number.

TAMs at different locations within the tumor may have different impacts on GC progression [14]. TAMs infiltration into tumor stroma has significant clinical relevance in GC, indicating the importance of not only studying the number but also studying the locations [15]. TAMs infiltration at invasive front could influence cancer metastasis through epithelial-mesenchymal transition (EMT) mechanism [16]. In turn, the degree of cell-to-cell contact may also influence the balance between protumoral and antitumoral properties of macrophages [17]. Some studies have also suggested that GC TAMs in different locations play different roles in relation to angiogenesis, stromal reaction, and prognosis [18]. Taken together, these results indicate that TAMs number and distributions are crucial factors to impact the coevolution between cancer cells and TAMs.

Therefore, this study evaluated both the number and locations of TAMs, especially the distribution pattern. TAMs number was counted at three locations, including cancer nest (MC), invasive front (MF), and stroma (MS). By comparison of TAMs number, TAMs distribution pattern could be defined as MC and MF/MS dominant pattern. Correlations of TAMs number and distribution pattern with GC clinical outcomes were both evaluated.

\section{RESULTS}

\section{Infiltrating macrophages in peritumoral and GC tissues}

Results were obtained according to the flowchart in Figure 1. Infiltrating macrophages could be seen in both peritumoral (Figure 2A) and GC tissues (Figure 2B). The median number of infiltrating macrophages in peritumoral tissues $(n=237)$ vs. GC tissues $(n=494)$ was 5.5 (range: 0-92.0) vs. 21.0 (range: 0-171.3) per high power field (hpf) $(P<0.001)$ (Figure 2C).

Among 204 GC cases in which TAMs presented both in cancer and peritumoral tissues, there was a trend towards significant higher number of TAMs in tumor tissues than peritumoral tissues $(P<0.001)$ (Figure 2D).

\section{Correlations between total TAMs number and clinic-pathological features and $\mathrm{OS}$}

The relationship between total TAMs number and major clinic-pathological characteristics were studied in 494 GC patients (Table 1). Total TAMs number was significantly correlated with pathological types $(P$ $=0.003)$, serosa invasion $(P=0.007)$ and TNM stage $(P$ $=0.009$ ), but not significantly correlated with age, gender, tumor location, histological grade, lymph node metastasis, distant metastasis $(P>0.05$ for all).

The median OS of $494 \mathrm{GC}$ cases was $21.0(95 \% \mathrm{CI}$ : 25.2-28.7) months (Figure 3A). There were 199 (40.3\%) dead, and the median OS was 15.0 (95CI: 16.6-20.3) months. Total TAMs number in survival group (median: 22.8, range: 0-157.5) was significantly higher than that in dead group (median: 17.5, range: 0-171.3) $(P<0.001)$. According to the median value of total TAMs number, 494 patients could be classified into total TAMs -low $(n=247)$ and -high subgroups $(n=247)$. The difference in OS between two subgroups was not statistically significant $(P=0.280)$ (Figure 3B and 3C).

\section{TAMs locations and distribution pattern}

According to the criteria on TAMs locations, 494 patients could be evaluated for MC, 319 patients could be evaluated for both MC and MF, and 296 patients could be evaluated for all MC, MF and MS. Major clinicpathological and survival information among the three databases were comparable (Supplementary Table 1). Detailed analyses were performed on 296 patients to study the impact of MC number, MF/MS number, and TAMs distribution pattern on GC prognosis.

The flowchart and detailed exclusion criteria was shown in Figure 4A. Representative photos of MC, MF, and MS were shown in Figure 4B-4D.

\section{Correlations between MC, MF/MS number and clinic-pathological features and $O S$}

Out of 296 GC patients, correlations between total TAMs number, MC number, MF/MS number, and major clinic-pathological characteristics were studied (Table 2). The median value of total TAMs number was 25 (range: 0 -171). Total TAMs number was significantly correlated with serosa invasion $(P=0.019)$, but not significantly correlated with age, gender, tumor location, histological grade, lymph node metastasis, distant metastasis, and TNM stage $(P>0.05$ for all). MC number was significantly correlated with tumor location $(P=0.018)$, and histological grade $(P=0.007)$, but not significantly correlated with age, gender, serosa invasion, lymph node metastasis, distant metastasis $(P>0.05$ for all). MF/MS number (median value: 13, range: 0-166) was higher than MC number (median value: 6, range: 0-126), the difference was statistically significant $(P<0.001) . \mathrm{MF} /$ MS number was significantly correlated with histological grade $(P=0.045)$, and lymph node metastasis $(P=0.037)$, but not significantly correlated with age, gender, tumor location, serosa invasion, distant metastasis, and TNM stage $(P>0.05$ for all). 
Correlations between total TAMs number, MC number, MF/MS number and GC OS were also investigated. According to the median value of macrophages number, 296 patients were classified as total TAMs -low $(\mathrm{n}=150)$ and -high $(\mathrm{n}=146)$, as MC -low (n $=149)$ and -high $(\mathrm{n}=147)$, as MF/MS -low $(\mathrm{n}=150)$ and -high ( $\mathrm{n}=146)$. No significant survival differences were observed regarding total TAMs number $(P=0.583)$, MC number $(P=0.544)$ and $\mathrm{MF} / \mathrm{MS}$ number $(P=0.104)$.

\section{Correlations between TAMs distribution pattern and clinic-pathological features and OS}

Among 296 GC patients, there were 128 (43.3\%) cases in which MC number (median: 12, range: 0-126) was significantly higher than MF/MS number (median: 4, range: 0 -75) $(P<0.001)$. These cases were defined as MC dominant pattern. Similarly, there were 168 (56.7\%) cases in which MF/MS number (median: 23, range: 1-166) was significantly higher than MC number (median: 4, range: 0-73) $(P<0.001)$. These patients were defined as MF/MS dominant pattern.
Compared with MC dominant pattern, MF/ MS dominant pattern was significantly correlated with histological grade $(P=0.001)$, pathological types $(P=0.004)$, but not significantly correlated with age, gender, tumor location, serosa invasion, lymph node metastasis, distant metastasis and TNM stage $(P>0.05$ for all) (Table 2). The median OS of MF/MS dominant pattern (22.1, 95\%CI: 23.5-28.9) was significantly shorter than that of MC dominant pattern (25.6, 95\%CI: 28.5-35.6) $(P$ $=0.002$, Figure $5 \mathrm{~A}$ ).

\section{Uni- and multivariate analysis and $\mathrm{ROC}$ analysis of TAMs distribution pattern}

The univariate analysis of clinic-pathological factors and TAMs distribution pattern regarding OS was also conducted. In univariate analysis, traditional clinicpathological features (such as histological grade, pT stage, $\mathrm{pN}$ stage and TNM stage), and MF/MS distribution pattern $(P=0.002)$ were associated with OS (Table 3$)$.

These factors were also integrated into multivariate Cox proportional hazards analysis. In multivariate
HE image

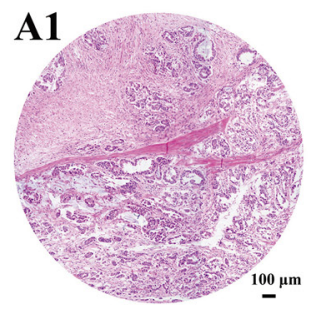

B1

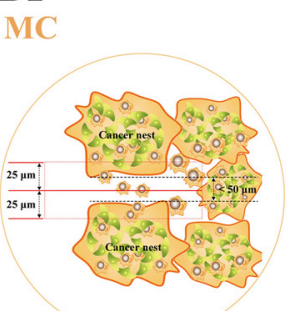

HE section

A2

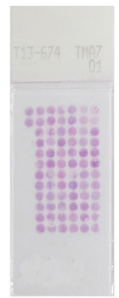

B2

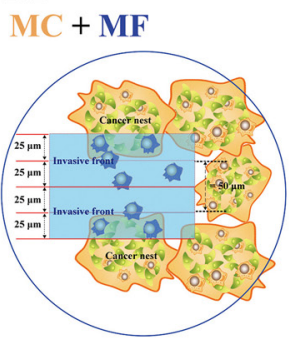

\section{TMAs IHC section IHC image}

A3

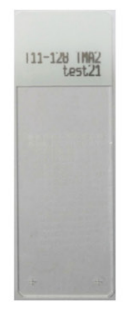

B3

$\mathrm{MC}+\mathrm{MF}+\mathrm{MS}$

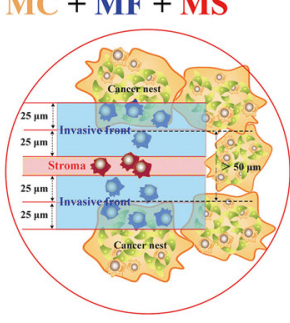

A4

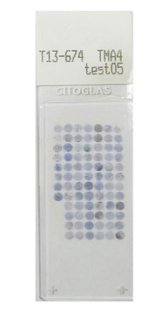

\section{A5}

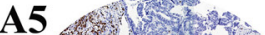

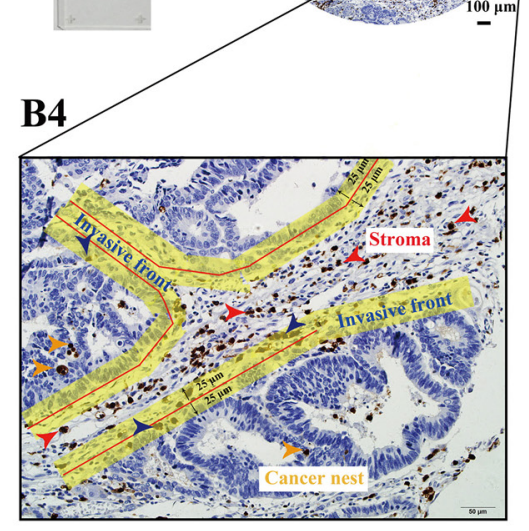

cancer cell

cancer nest

$\mathrm{MC}$

MF MS

Figure 1: Major technical procedures and definitions of TAMs distribution patterns. (A) Representative GC tissues were obtained for the establishment of tissue microarrays (TMAs) (A1-A3), and the immunohistochemistry experiments were performed well on TMAs (A4-A5). (B) The number and distribution of TAMs were analyzed and three TAMs locations including MC, MF, MS were found out. In virtual, one field could be divided into three parts, including cancer nest, cancer invasive front, and cancer stroma. The number of TAMs was evaluated for all locations. (B1) When the distance between two cancer nests were less than $50 \mu \mathrm{m}$, such two cancer nests were considered just one big cancer nest. The TAMs in those areas were named as MC, MC=Total TAMs. (B2) The TAMs in stroma and nest which stay from the junction of stroma and nest less or equal to $25 \mu \mathrm{m}$ were named as MF. When the distance between two cancer nests were just equal to $50 \mu \mathrm{m}$, there were only $\mathrm{MC}$ and $\mathrm{MF}, \mathrm{MC}+\mathrm{MF}=$ Total TAMs. (B3) When the distance between two cancer nests were more than $50 \mu \mathrm{m}$, such two cancer nests were considered as two distinct cancer nests. The TAMs in stromal which stay from the neighboring cancer nest more than $25 \mu \mathrm{m}$ were named as MS. MC+MF+MS =Total TAMs. (B4) In this study, there were 296 patients could evaluate MC, MF and MS simultaneously. 
analysis, significant factors correlating with $\mathrm{OS}$ were $\mathrm{pN}$ stage $(P=0.008)$, pM stage $(P=0.020)$, TNM stage $(P$ $<0.001$ ), and TAMs distribution pattern (hazard ratio, HR $=2.177$ [95\%CI: 1.449-3.270], $P<0.001)$ (Table 4).

ROC analysis was conducted to further evaluate the prognostic performance of histological grade, $\mathrm{pT}$ stage, $\mathrm{pN}$ stage, $\mathrm{pM}$ stage, TNM stage, and MF/MS distribution pattern. As shown in Figure 5B, the area under the curve (AUC) was the largest for TNM stage $[\mathrm{AUC}=0.676$, 95\%CI: $0.615-0.737](P<0.001)$, followed by $\mathrm{pN}$ stage [AUC $=0.653,95 \% \mathrm{CI}: 0.591-0.716](P<0.001), \mathrm{pT}$ stage [AUC $=0.587,95 \% \mathrm{CI}: 0.522-0.652](P=0.012)$, TAMs distribution pattern [AUC $=0.581,95 \% \mathrm{CI}: 0.514-0.647]$ $(P=0.020), \mathrm{pM}$ stage $[\mathrm{AUC}=0.543,95 \% \mathrm{CI}: 0.474-0.611]$ $(P=0.216)$, and histological grade $[\mathrm{AUC}=0.505,95 \% \mathrm{CI}$ : $0.438-0.573](P=0.876)$.

\section{DISCUSSION}

Inflammation is one of the significant hallmarks of cancer, and influences the coevolution between cancer cells and TME [19]. Within the TME, TAMs recruited by tumor-derived signals might be the most important inflammation cells [20,21]. Evaluating both the number and distributions could promote a comprehensive understanding of TAMs significance during GC progression.

In this study, TAMs number in GC tissues was much higher than that in peritumoral tissues. This result, in accordance with some previous studies, proved that TAMs were recruited and aggregated within tumor tissues, then mutually interacted with tumor cells [22]. In fact, TAMs also correlated with tumor progression. TAMs number had been identified as an independent prognostic factor in several cancer types, such as breast cancer and colorectal cancer [23, 24]. Herein, total TAMs number was higher in GC patients with no serosa invasion, early TNM stages, and in survival patients, which indicated TAMs may be protective factors of GC. To investigate the exact prognostic significance of TAMs, detailed analyses were focused on total TAMs number and OS, and no correlations were found. Such contradictory results have also been reported previously $[25,26]$. Some studies considered TAMs as protumoral through induction of
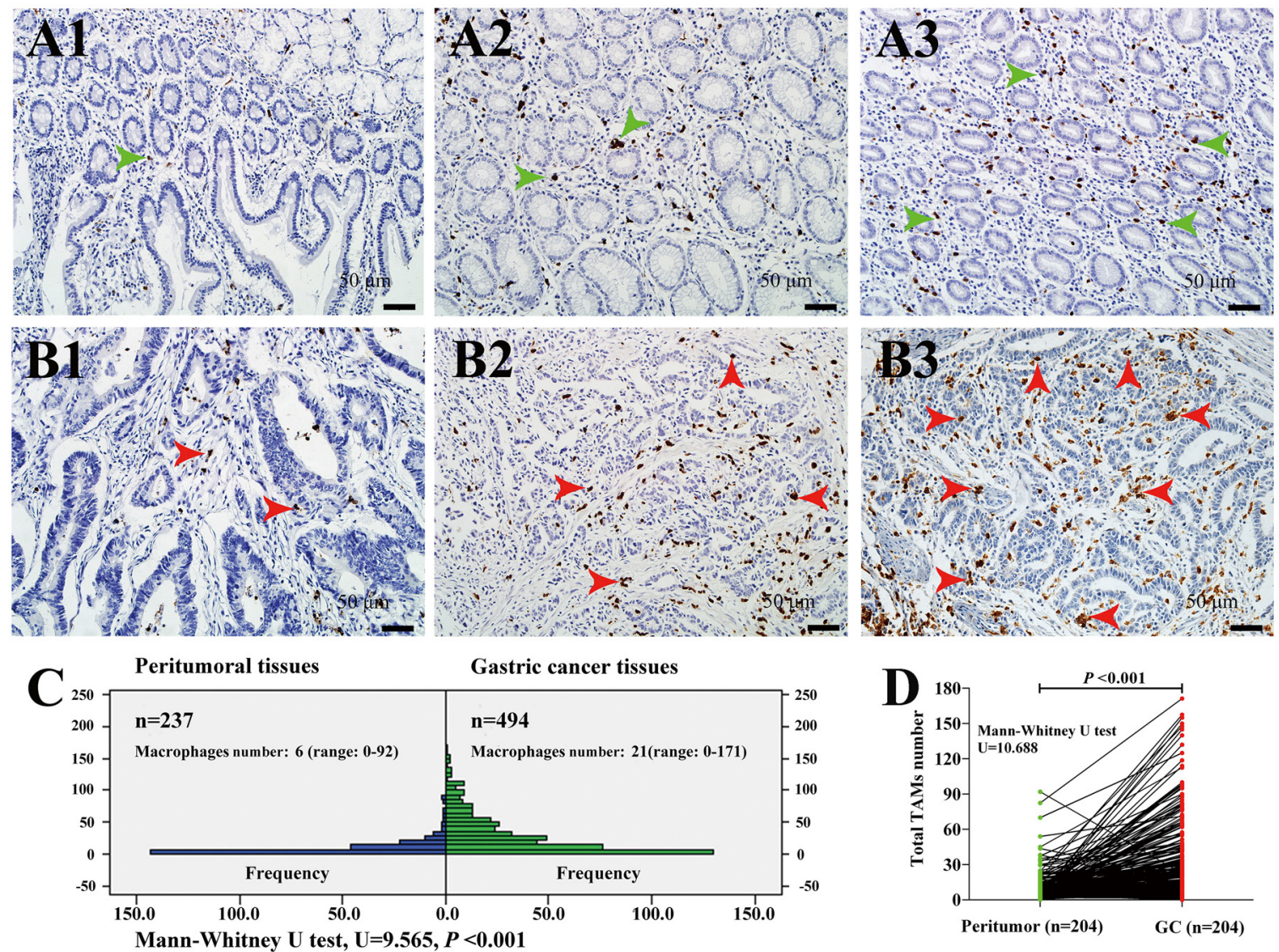

Figure 2: Infiltrating macrophages in peritumoral and GC tissues. (A) Infiltrating macrophages in peritumoral tissues gradually increased in A1, A2 and A3. Green arrowheads indicated macrophages. (B) Infiltrating macrophages in GC tissues. Macrophages were thinly scattered in B1, accumulated in B2 and in large numbers in B3. Red arrowheads indicated tumor associated macrophages (TAMs). (C) The frequency distribution of infiltrating macrophages number in 237 peritumoral tissues and 494 GC tissues. (D) The self-comparison of $204 \mathrm{GC}$ cases with relative peritumoral tissues. Magnification: $200 \times$, Scale bar $=50 \mu \mathrm{m}$. 


\begin{tabular}{|c|c|c|c|c|}
\hline \multirow{2}{*}{ Variables } & \multirow{2}{*}{ No. $(\%)$} & \multicolumn{3}{|c|}{ Total TAMs number } \\
\hline & & Median & Range & $P$ a \\
\hline Age (Means \pm SD, yrs) & $59.0 \pm 11.9$ & & & \\
\hline$\leq 59$ & $249(50.4)$ & 22 & $0-158$ & 0.896 \\
\hline$>59$ & $245(49.6)$ & 20 & $0-171$ & \\
\hline \multicolumn{5}{|l|}{ Gender } \\
\hline Male & $349(70.6)$ & 19 & $0-155$ & 0.073 \\
\hline Female & $145(29.4)$ & 24 & $0-171$ & \\
\hline \multicolumn{5}{|l|}{ Tumor location ${ }^{b}$} \\
\hline Distal stomach & $227(46.0)$ & 21 & $0-171$ & 0.965 \\
\hline Non-distal stomach & $267(54.0)$ & 20 & $0-158$ & \\
\hline \multicolumn{5}{|l|}{ Histological grade } \\
\hline $1 / 2$ & $164(33.2)$ & 22 & $0-145$ & 0.737 \\
\hline $3 / 4$ & $330(66.8)$ & 20 & $0-171$ & \\
\hline \multicolumn{5}{|l|}{ Pathological types } \\
\hline Well/Moderately differentiated adenocarcinoma & $131(26.5)$ & 23 & $0-145$ & 0.003 \\
\hline Low/Undifferentiated adenocarcinoma & $284(57.5)$ & 23 & $0-171$ & \\
\hline Mucinous adenocarcinoma/signet-ring cell carcinoma & $66(13.4)$ & 13 & $0-105$ & \\
\hline Others & $13(2.6)$ & 9 & $0-78$ & \\
\hline \multicolumn{5}{|l|}{ Serosa invasion } \\
\hline No $(\mathrm{T} 0, \mathrm{~T} 1, \mathrm{~T} 2)$ & $104(21.1)$ & 27 & $0-158$ & 0.007 \\
\hline Yes $(\mathrm{T} 3, \mathrm{~T} 4)$ & $390(78.9)$ & 18 & $0-171$ & \\
\hline \multicolumn{5}{|l|}{ Lymph node metastasis } \\
\hline No (N0) & $166(33.6)$ & 23 & $0-158$ & 0.472 \\
\hline Yes $(\mathrm{N} 1, \mathrm{~N} 2, \mathrm{~N} 3)$ & $328(66.4)$ & 19 & $0-171$ & \\
\hline \multicolumn{5}{|l|}{ Distant Metastasis } \\
\hline M0 & $473(95.7)$ & 21 & $0-171$ & 0.734 \\
\hline M1 & $21(4.3)$ & 31 & $0-126$ & \\
\hline \multicolumn{5}{|l|}{ TNM stages } \\
\hline Early (Stages I, II) & $177(35.8)$ & 26 & $0-155$ & 0.009 \\
\hline Advanced (Stages III, IV) & $317(64.2)$ & 16 & $0-171$ & \\
\hline \multicolumn{5}{|l|}{ Survive } \\
\hline Yes & $295(59.7)$ & 23 & $0-158$ & $<0.001$ \\
\hline No & $199(40.3)$ & 18 & $0-171$ & \\
\hline
\end{tabular}

a $P$-value in bold indicates that the difference was significant

b Tumor location was classified according to the Japanese classification of gastric carcinoma (3rd English edition) 

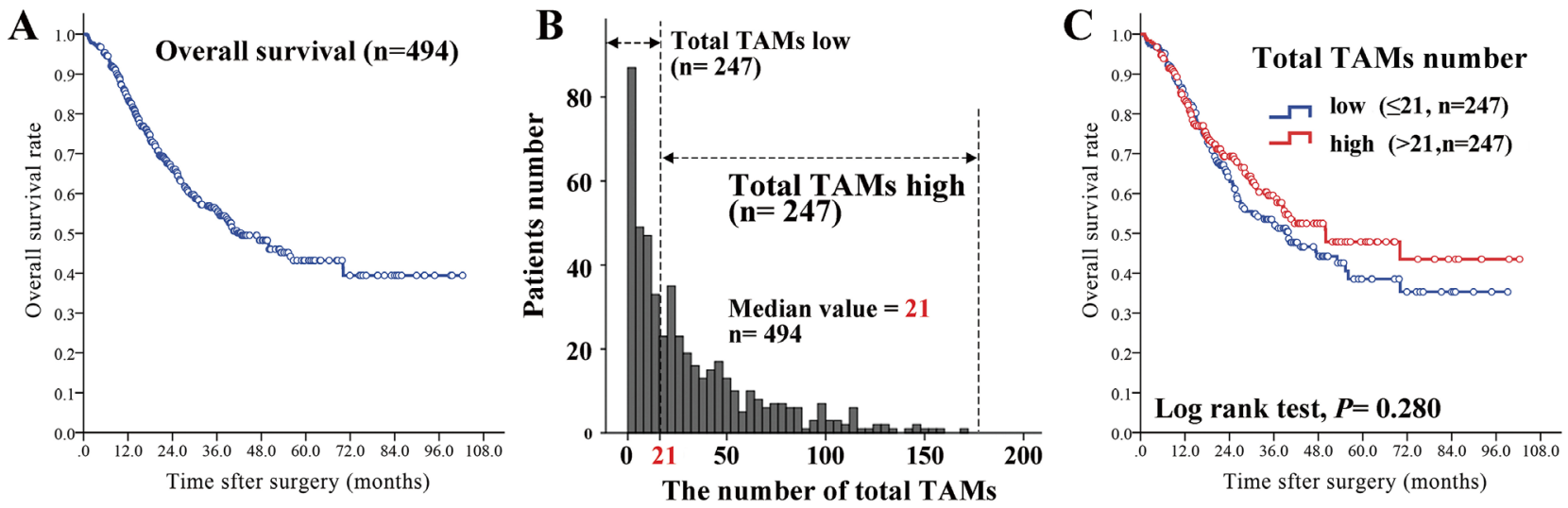

Figure 3: The prognostic value of total TAMs number. (A) The median OS of 494 GC cases was 21.0 (range: 0.77-102.33) months. (B) Using the median value of total TAMs number as the threshold, there were $247(50.0 \%)$ and $247(50.0 \%)$ patients documented as total TAMs low and high number subgroup. (C) There was no significant difference regarding GC OS between total TAMs low and high number subgroups.
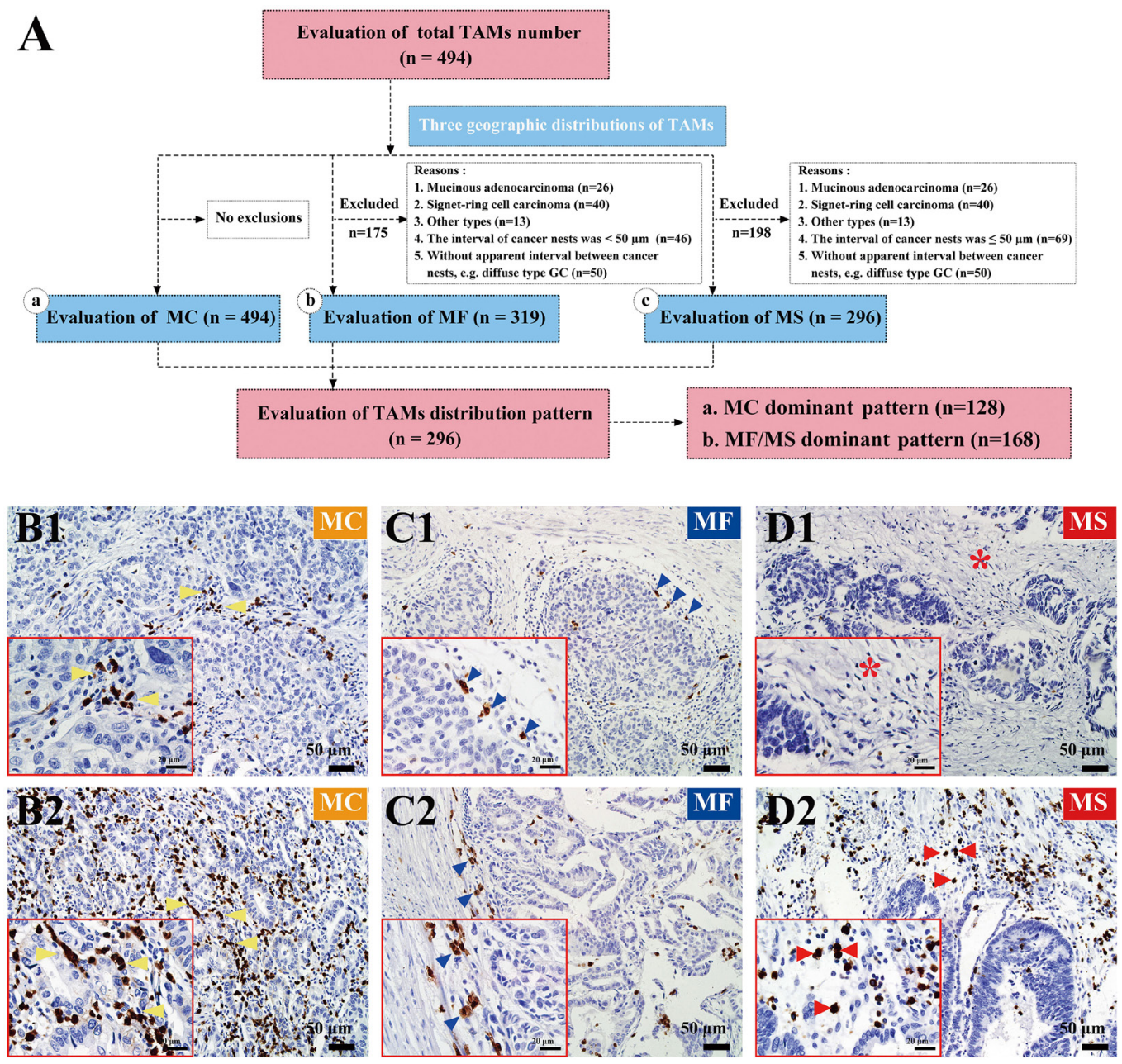

Figure 4: TAMs locations and distribution patterns. (A) The flowchart and detailed exclusion criteria. (B) MC were diffusely distributed within cancer nest. Tumor epithelium-infiltrating macrophages were representative features to present spatial relationships between TAMs and cancer cells. Yellow arrowheads in B1 and B2 indicated MC with low and high number, respectively. (C) Blue arrowheads in $\mathrm{C} 1$ and $\mathrm{C} 2$ indicated MF with low and high number, respectively. (D) MS were mostly seen in well and moderately differentiated adenocarcinoma. Red stars in D1 showed stromal regions with no TAMs infiltration. Red arrowheads in D2 indicated MS with high number. Magnification: $\times 200$, Scale bar $=50 \mu \mathrm{m}$. Photos in red frames, Magnification: $\times 400$, Scale bar $=20 \mu \mathrm{m}$. 
Table 2: Major clinic-pathological characteristics and TAMs number, distribution pattern in 296 GC patients

\begin{tabular}{|c|c|c|c|c|c|c|c|c|c|c|c|c|c|}
\hline \multirow[t]{2}{*}{ Variables } & \multirow{2}{*}{ No. (\%) } & \multicolumn{3}{|c|}{ Total TAMs number } & \multicolumn{3}{|c|}{ MC number } & \multicolumn{3}{|c|}{ MF/MS number } & \multicolumn{3}{|c|}{$\begin{array}{l}\text { TAMs distribution pattern } \\
(\mathbf{n}, \%)\end{array}$} \\
\hline & & Median & Range & $P^{\text {a }}$ & Median & Range & $P^{a}$ & Median & Range & $P^{a}$ & MC & MF/MS & $P^{a}$ \\
\hline Age (Means $\pm \mathrm{SD}$, yrs $)$ & $59.9 \pm 11.7$ & & & & & & & & & & & & \\
\hline$\leq 59$ & $145(49.0)$ & 28 & $0-171$ & 0.352 & 8 & $0-90$ & 0.201 & 12 & $0-160$ & 0.563 & $63(21.3)$ & $82(27.7)$ & 1.000 \\
\hline$>59$ & $151(51.0)$ & 22 & $0-171$ & & 5 & $0-126$ & & 14 & $0-160$ & & $65(22.0)$ & $86(29.0)$ & \\
\hline \multicolumn{14}{|l|}{ Gender } \\
\hline Male & $218(73.6)$ & 24 & $0-171$ & 0.513 & 6 & $0-95$ & 0.943 & 13 & $0-114$ & 0.359 & $92(31.1)$ & $126(42.5)$ & 0.595 \\
\hline Female & $78(26.4)$ & 26 & $1-171$ & & 6 & $0-126$ & & 18 & $0-166$ & & $36(12.2)$ & $42(14.2)$ & \\
\hline \multicolumn{14}{|l|}{ Tumor location ${ }^{b}$} \\
\hline Upper third & $95(32.1)$ & 23 & $0-171$ & 0.145 & 6 & $0-95$ & 0.018 & 14 & $0-110$ & 0.545 & $36(12.2)$ & 59 (19.9) & 0.473 \\
\hline Middle third & $75(25.3)$ & 30 & $1-166$ & & 11 & $0-90$ & & 16 & $0-166$ & & $37(12.5)$ & $38(12.8)$ & \\
\hline Lower third & $115(38.9)$ & 23 & $0-171$ & & 6 & $0-126$ & & 13 & $0-160$ & & $51(17.2)$ & $64(21.7)$ & \\
\hline Total stomach & $11(3.7)$ & 15 & $3-101$ & & 4 & $0-39$ & & 7 & $0-80$ & & $4(1.3)$ & $7(2.4)$ & \\
\hline \multicolumn{14}{|l|}{ Histological grade } \\
\hline $1 / 2$ & $124(41.9)$ & 25 & $0-136$ & 0.906 & 4 & $0-85$ & 0.007 & 19 & $0-120$ & 0.045 & $40(13.5)$ & $84(28.4)$ & 0.001 \\
\hline $3 / 4$ & $172(58.1)$ & 24 & $0-171$ & & 9 & $0-126$ & & 11 & $0-166$ & & $88(29.7)$ & $84(28.4)$ & \\
\hline \multicolumn{14}{|l|}{ Serosa invasion } \\
\hline No $(\mathrm{T} 0, \mathrm{~T} 1, \mathrm{~T} 2)$ & $68(23.0)$ & 31 & $1-171$ & 0.019 & 8 & $0-115$ & 0.582 & 20 & $0-160$ & 0.053 & $27(9.1)$ & 41 (13.9) & 0.577 \\
\hline Yes (T3, T4) & $228(77.0)$ & 21 & $0-171$ & & 6 & $0-126$ & & 12 & $0-166$ & & $101(34.2)$ & $127(42.8)$ & \\
\hline \multicolumn{14}{|l|}{ Lymph node metastasis } \\
\hline No (N0) & $100(33.8)$ & 29 & $0-171$ & 0.111 & 7 & $0-78$ & 0.806 & 16 & $0-110$ & 0.037 & $38(12.9)$ & $62(20.9)$ & 0.216 \\
\hline Yes (N1, N2, N3) & $196(66.2)$ & 22 & $0-171$ & & 6 & $0-126$ & & 11 & $0-166$ & & $90(30.4)$ & $106(35.8)$ & \\
\hline \multicolumn{14}{|l|}{ Distant Metastasis } \\
\hline M0 & $283(95.6)$ & 25 & $0-171$ & 0.783 & 7 & $0-126$ & 0.085 & 13 & $0-160$ & 0.783 & $123(41.6)$ & $160(54.0)$ & 0.782 \\
\hline M1 & $13(4.4)$ & 26 & $1-166$ & & 3 & $0-84$ & & 14 & $0-166$ & & $5(1.7)$ & $8(2.7)$ & \\
\hline \multicolumn{14}{|l|}{ TNM stage } \\
\hline Stages I & $45(15.2)$ & 30 & $2-104$ & 0.338 & 8 & $0-50$ & 0.296 & 18 & $0-100$ & 0.187 & $16(5.4)$ & $29(9.8)$ & 0.704 \\
\hline Stages II & $67(22.6)$ & 26 & $0-171$ & & 6 & $0-90$ & & 15 & $0-160$ & & $29(9.8)$ & $38(12.8)$ & \\
\hline Stages III & $172(58.1)$ & 20 & $0-171$ & & 7 & $0-126$ & & 11 & $0-120$ & & $78(26.3)$ & $94(31.8)$ & \\
\hline Stages IV & $12(4.1)$ & 25 & $1-166$ & & 3 & $0-84$ & & 11 & $0-166$ & & $5(1.7)$ & $7(2.4)$ & \\
\hline \multicolumn{14}{|l|}{ Survive } \\
\hline Yes & $182(61.5)$ & 25 & $0-171$ & 0.812 & 8 & $0-115$ & 0.284 & 13 & $0-120$ & 0.551 & $90(30.4)$ & $92(31.1)$ & 0.008 \\
\hline No & $114(38.5)$ & 22 & $0-171$ & & 5 & $0-126$ & & 14 & $0-166$ & & $38(12.8)$ & $76(25.7)$ & \\
\hline
\end{tabular}

a $P$-value in bold indicates that the difference was statistically significant

b Tumor location was classified according to the Japanese classification of gastric carcinoma (3rd English edition)

angiogenesis and suppression of antitumor immunity [27], while other reports found that a dense TAMs infiltration positively influenced prognosis in GC [9]. To explore such contradiction, we studied TAMs from aspects of both the number and histological distributions.

Based on the spatial position relationships between TAMs and tumor cells, MC and MF/MS number were counted and analyzed. The distribution characteristic of TAMs had also been mentioned previously [28, 29]. Su et al. elucidated mutual interactions between cancer cells and TAMs at tumor invasive front [28]. Additionally, the close vicinity of TAMs and tumor neo-vessels was observed in tumor stroma, and constituted tumor invasion unit [29]. On the basis of these morphological studies, quantitative 
Table 3: Analyses of clinic-pathological factors and TAMs distribution pattern regarding OS in 296 GC patients

\begin{tabular}{|c|c|c|c|c|c|c|}
\hline Variables & No. $(\%)$ & No. of death $(\%)$ & $\begin{array}{c}\text { 3-year survival } \\
\text { rate }\end{array}$ & $\begin{array}{c}\text { 5-year survival } \\
\text { rate }\end{array}$ & $\begin{array}{c}\text { Log-rank test } \chi^{2} \\
\text { value }\end{array}$ & $\boldsymbol{P}^{*}$ \\
\hline \multicolumn{7}{|l|}{ Histological grade } \\
\hline $1 / 2$ & $124(41.9)$ & $47(37.9)$ & $57.36 \%$ & $45.52 \%$ & 0.104 & 0.747 \\
\hline $3 / 4$ & $172(58.1)$ & $67(39.0)$ & $57.80 \%$ & $44.88 \%$ & & \\
\hline \multicolumn{7}{|l|}{ T stage } \\
\hline $\mathrm{T} 1-2$ & $68(23.0)$ & $14(20.6)$ & $71.06 \%$ & $66.62 \%$ & 8.727 & 0.003 \\
\hline T3-4 & $228(77.0)$ & $100(43.9)$ & $54.02 \%$ & $39.86 \%$ & & \\
\hline \multicolumn{7}{|l|}{ Lymph nodes metastasis } \\
\hline No & $100(33.8)$ & $17(17.0)$ & $76.00 \%$ & $73.14 \%$ & 25.330 & $<0.001$ \\
\hline Yes & $196(66.2)$ & $97(49.5)$ & $48.98 \%$ & $33.00 \%$ & & \\
\hline \multicolumn{7}{|l|}{ Distant Metastasis } \\
\hline M0 & $283(95.6)$ & $103(36.4)$ & $60.01 \%$ & $46.90 \%$ & 21.956 & $<0.001$ \\
\hline M1 & $13(4.4)$ & $11(84.6)$ & $13.19 \%$ & $13.19 \%$ & & \\
\hline \multicolumn{7}{|l|}{ TNM stage } \\
\hline Stages I & $45(15.2)$ & $6(13.3)$ & $78.94 \%$ & $78.94 \%$ & 37.833 & $<0.001$ \\
\hline Stages II & $67(22.6)$ & $16(23.9)$ & $71.70 \%$ & $63.00 \%$ & & \\
\hline Stages III & $172(58.1)$ & $82(47.7)$ & $50.90 \%$ & $34.90 \%$ & & \\
\hline Stages IV & $12(4.1)$ & $10(83.3)$ & $14.29 \%$ & $14.29 \%$ & & \\
\hline TAMs distribution pattern & $296(100)$ & $114(38.5)$ & $57.60 \%$ & $45.06 \%$ & 9.722 & 0.002 \\
\hline MC dominant pattern & $128(43.2)$ & $38(29.7)$ & $68.21 \%$ & $53.24 \%$ & & \\
\hline MF/MS dominant pattern & $168(56.8)$ & $76(45.2)$ & $49.85 \%$ & $38.78 \%$ & & \\
\hline
\end{tabular}

*: $P$-value in bold indicates that the difference was significant.
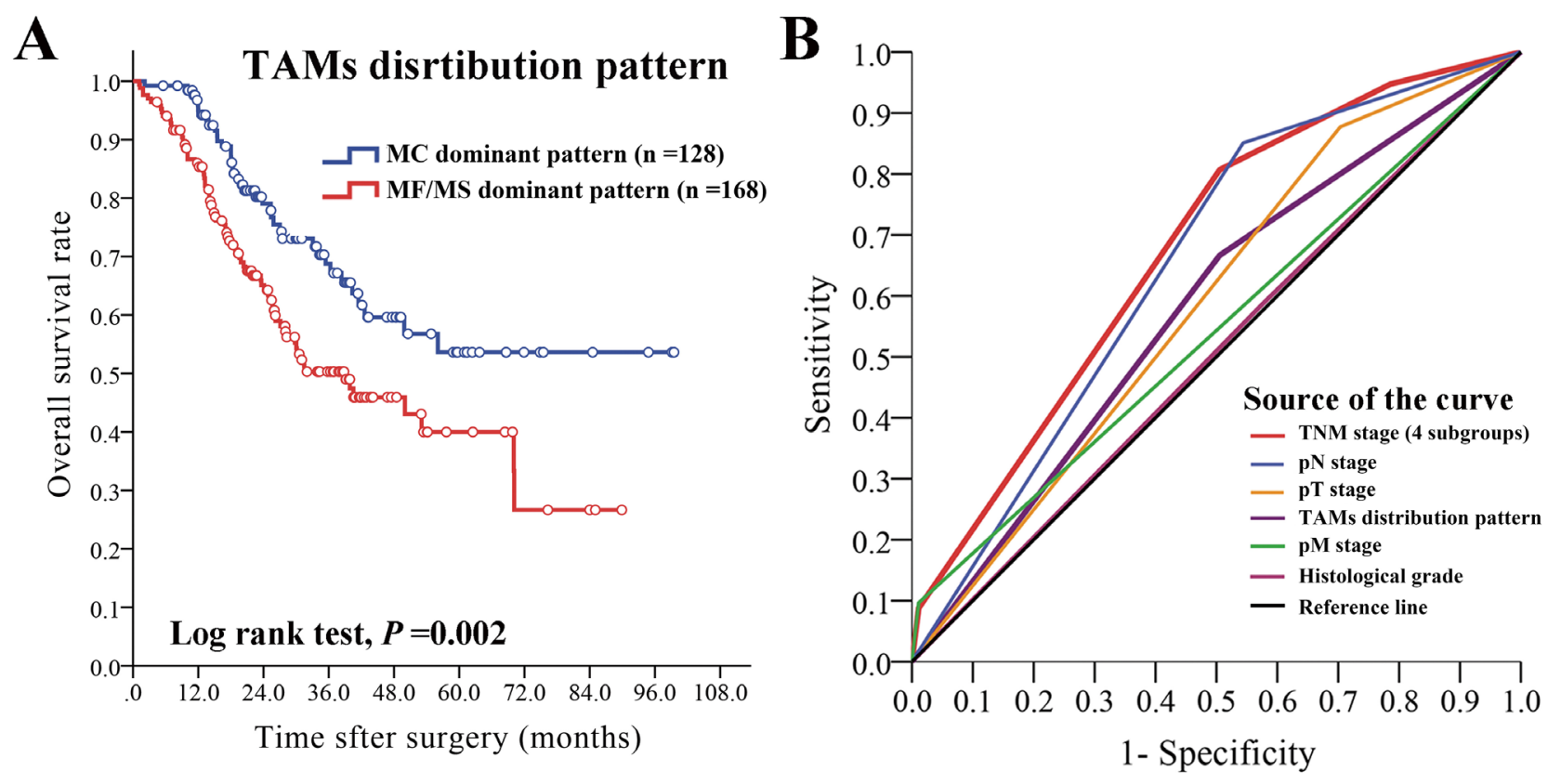

Figure 5: The prognostic value of TAMs distribution pattern and ROC analysis. (A) The prognostic value of TAMs distribution pattern. The median OS of patients with MF/MS dominant pattern was shorter than that of patients with MC dominant pattern, the differences was statistically significant $(P=0.002)$. (B) ROC analysis indicated that TAMs distribution pattern (AUC: 0.581 [95\%CI: 0.514-0.647], $P=0.020$ ) was superior to histological grade and $\mathrm{pM}$ stage, but inferior to $\mathrm{pN}$ stage and TNM stage. 
Table 4: Multivariate analysis and ROC analysis of factors associated with OS

\begin{tabular}{|c|c|c|c|c|c|c|}
\hline \multirow[b]{2}{*}{ Factors } & \multicolumn{3}{|c|}{ OS } & \multicolumn{3}{|c|}{ ROC analysis } \\
\hline & HR & $95 \% \mathrm{CI}$ & $P^{*}$ & $\begin{array}{l}\text { Area under } \\
\text { curve (AUC) }\end{array}$ & $95 \%$ CI & $\boldsymbol{P}^{*}$ \\
\hline Histological grade & & & & 0.505 & $0.438-0.573$ & 0.876 \\
\hline $1 / 2$ & 1.000 & & & & & \\
\hline $3 / 4$ & 0.971 & $0.661-1.436$ & 0.894 & & & \\
\hline T stage & & & & 0.587 & $0.522-0.652$ & 0.012 \\
\hline $\mathrm{T} 0,1,2$ & 1.000 & & 0.071 & & & \\
\hline $\mathrm{T} 3,4$ & 3.352 & $0.903-12.437$ & & & & \\
\hline N stage & & & & 0.653 & $0.591-0.716$ & $<0.001$ \\
\hline N0 & 1.000 & & 0.008 & & & \\
\hline $\mathrm{N} 1,2,3$ & 6.073 & $1.590-23.192$ & & & & \\
\hline M stage & & & & 0.543 & $0.474-0.611$ & 0.216 \\
\hline M0 & 1.000 & & 0.020 & & & \\
\hline M1 & 17.034 & $1.568-185.083$ & & & & \\
\hline TNM stage & & & & 0.676 & $0.615-0.737$ & $<0.001$ \\
\hline Stage I & 1.000 & & & & & \\
\hline Stage II & 1.677 & $0.654-4.297$ & 0.281 & & & \\
\hline Stage III & 4.007 & $1.746-9.199$ & 0.001 & & & \\
\hline Stage IV & 10.880 & $3.929-30.130$ & $<0.001$ & & & \\
\hline $\begin{array}{l}\text { TAMs distribution } \\
\text { pattern }\end{array}$ & & & & 0.581 & $0.514-0.647$ & 0.020 \\
\hline MC dominant & 1.000 & & & & & \\
\hline MF/MS dominant & 2.177 & $1.449-3.270$ & $<0.001$ & & & \\
\hline
\end{tabular}

*: $P$-value in bold indicates that the difference was significant.

counting analyses of TAMs were further conducted among different sites in GC. It turned out that MF/MS number was much higher than MC number, showing that TAMs tended to locate at invasive front and in stroma. However, no significant relationships could be reported between total TAMs number, MC number or MF/MS number and GC prognosis in this study. Therefore, simply analyzing TAMs number with ignorance of distribution locations was not sufficient [30]. Taken together, TAMs number might not be a significant prognostic marker related to GC OS.

Herein further analysis was developed using TAMs distribution pattern through a definition by systematical integration of TAMs number and histological distributions, which is crucial to reflect the coevolution between tumor cells and TME [31]. In this study, GC patients with
MF/MS dominant pattern had worse clinical outcomes than $\mathrm{MC}$ dominant pattern, indicating $\mathrm{MF} / \mathrm{MS}$ as main contributors to promote GC progression. Moreover, the predictive value of TAMs distribution pattern was verified by ROC analysis. Theoretically, the heterogeneity and plasticity were hallmarks of macrophages [32]. TAMs could undergo phenotypic switch from the classically activated macrophages (also known as M1 macrophages) to the alternatively activated macrophages (M2 macrophages). M1 macrophages were described as pro-inflammatory to display tumor-resistant effects, while M2 macrophages often associated with tumor-promoting properties. Consequently, TAMs gained potentials to promote cancer cell motility in invasion areas, to promote metastasis in stromal and perivascular areas, and to 
stimulate angiogenesis in avascular and peri-necrotic hypoxic areas [33]. Therefore, TAMs among different sites in GC tissues might represent distinct significances and prognostic values [34]. Owing to vigorous cell-to-cell contacts among TAMs, cancer cells and other activated stromal cells, TAMs along tumor invasive front are of great significance. At tumor invasive front, TAMs might correlate with various signaling pathways [35], undergo phenotypically switch from M1 to M2 [36], and finally promote the acquisition of specific pathological features of cancers such as immunosuppression, neovascularization and modification of extracellular matrix [37]. Pinto et al. have shown that TAMs with M2 phenotype were recruited by multiple chemoattractant and accumulated at the interface between cancer nest and stroma [38], thereby raising a hypothesis that the main constituent of $\mathrm{MF} / \mathrm{MS}$ was M2 macrophages, which had been verified in some studies and correlated with poor prognosis [39].

With these in consideration, MF/MS could be a predominant and decisive factor, while MC might be a less important factor on GC prognosis. TAMs distribution pattern integrated both $\mathrm{MC}$ and $\mathrm{MF} / \mathrm{MS}$ into consideration, and could be an independent prognostic factor of GC. The advantage of TAMs distribution pattern was due to the significance of researching TME and complex quantitative analyses [40]. However, limitations also existed by optimizing single biomarker, thus demonstrating TAMs distribution pattern inferior to TNM stage. To make sense of the overall landscape of TAMs, combined analyses with other biomarkers may provide new insights for deep understandings [41].

In summary, TAMs number merely correlates with several unfavorable clinic-pathological features, but has no significant prognostic value. Both the number and distributions should be taken into consideration for TAMs evaluations. Promisingly, TAMs distribution pattern could be a new factor related to GC OS.

\section{MATERIALS AND METHODS}

\section{Study population and database}

The records of patients who underwent surgical resection of GC from December 2002 to February 2011 were reviewed. Major demographic and clinicpathological characteristics were retrieved. The tumor type, histologic grade, depth of invasion, number of lymph nodes retrieved, and number of lymph nodes with metastases were re-confirmed histologically. Inclusion and exclusion criteria were defined as follows. Patients were included when histology confirmed adenocarcinoma of the stomach and the survival data were available. Patients were excluded when distant metastasis has been diagnosed before surgery, histology identified a pathological type other than adenocarcinoma, R1 resection, no lymph node was retrieved or histopathological and survival data were incomplete. No patients receive neoadjuvant chemotherapy. In this study, $329(66.6 \%)$ patients received adjuvant chemotherapy. In stage III patients $(n=316), 217$ $(68.7 \%)$ patients received adjuvant chemotherapy, and only 69 patients received more than six cycles of adjuvant chemotherapy. TNM stage was determined according to the 7 th edition UICC/AJCC TNM staging system. Overall survival (OS), defined as the duration from operation to GC-related death or last follow-up, was used for prognosis evaluation. The primary endpoint of this study was OS, and patients alive at the last follow-up were recorded as censored events.

\section{Ethics statement}

Written informed consent was obtained from the patients with the study protocol approved by the ethics committee of Zhongnan Hospital of Wuhan University. The study was undertaken in accordance with the ethical standards of the World Medical Association Declaration of Helsinki.

\section{Tissue microarrays (TMAs) and immunohistochemistry (IHC)}

TMAs have been constructed for this study. Briefly, two cores were taken from each representative tumor tissue and peritumoral tissue (at least $50 \mathrm{~mm}$ away from the tumor border). Then, sixteen TMAs sections with 494 tumor tissues (988 cores, $2 \mathrm{~mm}$ each core) and 237 peritumoral tissues (474 cores, $2 \mathrm{~mm}$ each core) were constructed (in collaboration with Shanghai Biochip Company Ltd., Shanghai, China).

Routine IHC method was performed for the staining of TAMs. The primary antibody was mouse anti-human monoclonal antibody against macrophages (ab22506 [MAC387], Abcam, UK, dilution 1/100), with corresponding horseradish peroxidase (HRP) conjugated secondary antibody (ab97265, Abcam, UK, dilution $1 / 300)$. The reaction products were visualized with diaminobenzidine (DAB, DAKO, Denmark). Then the slides were evaluated by two senior pathologists, who were blinded to the patients' clinical features and outcomes. A consensus was achieved using a multi-headed microscope in case of discrepancy. In brief, one TMA core with at least 4 standard-compliant vision fields (magnification, $\times 200$ ) per patient was considered to be adequate, with no focus on hotspots.

\section{Image acquisition and the classification criteria of TAMs locations and distribution patterns}

The digital images of infiltrating macrophages staining were captured under Olympus BX51 fluorescence microscope equipped with Olympus DP72 camera (Olympus Optical Co., Ltd., Tokyo, Japan) at $\times 200$ 
magnification. Identical settings were used for every photograph, so as to minimize the selection bias.

To assess the role of TAMs in GC progression, the number and the distribution of TAMs were evaluated. First, the positive cells were counted in at least four high power fields (hpfs, $\times 200$ magnification) each core, and the average number of two cores (eight hpfs) from the same patient were recorded as the number of TAMs. Second, TAMs geographic distributions were assessed, aiming to uncover the exact role of TAMs in different areas. Theoretically, each field could be divided into three different regions including cancer nest, cancer invasive front and cancer stromal. Correspondingly, the TAMs in GCs could be classified into three distinct patterns including infiltrating macrophages in cancer nest (MC), infiltrating macrophages in invasive front (MF) and infiltrating macrophages in stromal (MS). Such classification represented the spatial position relationship between macrophages and cancer cells.

Then, the number of MC, MF and MS were recorded, respectively. Briefly, $\mathrm{MC}+\mathrm{MF}+\mathrm{MS}=$ Total TAMs. Notable, MF and MS may be zero. Actually, not each patient has three locations simultaneously, but may have one superiority location. Thus, some patients may have $\mathrm{MC}$ only, some patients may have $\mathrm{MC}$ and $\mathrm{MF}$, and some patients may have MC, MF and MS simultaneously. In this study, there were 494 patients could be evaluated for MC, 319 patients could be evaluated for both MC and MF, and only 296 patients could be evaluated for all MC, MF and MS. Finally, total of 296 patients containing three locations of TAMs were classified into two distribution patterns, including MC dominant pattern and MF/MS dominant pattern. Investigators were blind to the clinicpathological data and clinical outcomes of GC patients. Cut points of TAMs number for subgroup analysis were explored by the median value.

\section{Statistical analysis}

Statistical analyses were performed with IBM SPSS 19.0 software (SPSS Institute, Chicago, IL). The correlations between the number of infiltrating TAMs and clinic-pathological parameters were calculated with the chi-square test. Survival probabilities between subgroups were analyzed with the Kaplan-Meier method, by use of the log-rank test for univariate analyses, and by use of Cox regression model for multivariate analyses. ROC analysis was used to determine the predictive value of the parameters. Two-sided $P$ value of $<0.05$ was considered to be statistically significant.

\section{Abbreviations}

GC, gastric cancer; TME, tumor microenvironment; TAMs, tumor associated macrophages; IHC, immunohistochemistry; MC, macrophages in cancer nest;
$\mathrm{MF}$, macrophages in invasive front; MS, macrophages in stroma; OS, overall survival; HR, hazard ratio; CI, confidence interval; ROC, receiver operating characteristic curve; AUC, area under the curve.

\section{FUNDING}

This work was supported by Science Fund of the National Natural Science Foundation of China (No. $81401515 ; 81230031$ ), and the Fundamental Research Funds for the Central Universities of Ministry of Education of China (No. 2042014kf0096). This work was also funded by "351 talent project (Luojia Young Scholars)" of Wuhan University.

\section{CONFLICTS OF INTEREST}

The authors declare no conflicts of interest.

\section{REFERENCES}

1. Turley SJ, Cremasco V, Astarita JL. Immunological hallmarks of stromal cells in the tumour microenvironment. Nat Rev Immunol. 2015; 15:669-82. https://doi. org/10.1038/nri3902.

2. Zhan HX, Zhou B, Cheng YG, Xu JW, Wang L, Zhang GY, $\mathrm{Hu}$ SY. Crosstalk between stromal cells and cancer cells in pancreatic cancer: New insights into stromal biology. Cancer Lett. 2017; 392:83-93. https://doi.org/10.1016/j. canlet.2017.01.041.

3. Gentles AJ, Newman AM, Liu CL, Bratman SV, Feng W, Kim D, Nair VS, Xu Y, Khuong A, Hoang CD, Diehn M, West RB, Plevritis SK, et al. The prognostic landscape of genes and infiltrating immune cells across human cancers. Nat Med. 2015; 21:938-45. https://doi.org/10.1038/ nm.3909.

4. Szebeni GJ, Vizler C, Kitajka K, Puskas LG. Inflammation and cancer: extra- and intracellular determinants of tumor-associated macrophages as tumor promoters. Mediators Inflamm. 2017; 2017:9294018. https://doi. org/10.1155/2017/9294018.

5. Hu W, Li X, Zhang C, Yang Y, Jiang J, Wu C. Tumorassociated macrophages in cancers. Clin Transl Oncol. 2016; 18:251-8. https://doi.org/10.1007/s12094-015-1373-0.

6. Galon J, Costes A, Sanchez-Cabo F, Kirilovsky A, Mlecnik $\mathrm{B}$, Lagorce-Pagès $\mathrm{C}$, Tosolini $\mathrm{M}$, Camus $\mathrm{M}$, Berger A, Wind $\mathrm{P}$, Zinzindohoué F, Bruneval $\mathrm{P}$, Cugnenc $\mathrm{PH}$, et al. Type, density, and location of immune cells within human colorectal tumors predict clinical outcome. Science. 2006; 313:1960-4. https://doi.org/10.1126/science.1129139.

7. Zhang J, Yan Y, Yang Y, Wang L, Li M, Wang J, Liu X, Duan X, Wang J. High infiltration of tumor-associated macrophages influences poor prognosis in human gastric cancer patients, associates with the phenomenon of 
EMT. Medicine (Baltimore). 2016; 95:e2636. https://doi. org/10.1097/MD.033712R1033712R12636.

8. Zhang WJ, Zhou ZH, Guo M, Yang LQ, Xu YY, Pang TH, Gao ST, Xu XY, Sun Q, Feng M, Wang H, Lu CL, $\mathrm{Wu}$ GZ, et al. High infiltration of polarized CD163+ tumor-associated macrophages correlates with aberrant expressions of CSCs markers, and predicts prognosis in patients with recurrent gastric cancer. J Cancer. 2017; 8:363-70. https://doi.org/10.7150/jca.16730.eCollection 2017.

9. Wang B, Xu D, Yu X, Ding T, Rao H, Zhan Y, Zheng L, Li L. Association of intra-tumoral infiltrating macrophages and regulatory $\mathrm{T}$ cells is an independent prognostic factor in gastric cancer after radical resection. Ann Surg Oncol. 2011; 18:2585-93. https://doi.org/10.1245/s10434-011-1609-3.

10. Li J, Liao Y, Ding T, Wang B, Yu X, Chu Y, Xu J, Zheng L. Tumor-infiltrating macrophages express interleukin-25 and predict a favorable prognosis in patients with gastric cancer after radical resection. Oncotarget. 2016; 7:1108393. https://doi.org/10.18632/oncotarget.7095.

11. Strachan DC, Ruffell B, Oei Y, Bissell MJ, Coussens LM, Pryer N, Daniel D. CSF1R inhibition delays cervical and mammary tumor growth in murine models by attenuating the turnover of tumor-associated macrophages and enhancing infiltration by CD8+ T cells. Oncoimmunology. 2013; 2:e26968. https://doi.org/10.4161/onci.26968

12. Yamaguchi T, Fushida S, Yamamoto $\mathrm{Y}$, Tsukada T, Kinoshita J, Oyama K, Miyashita T, Tajima H, Ninomiya I, Munesue S, Harashima A, Harada S, Yamamoto H, et al. Tumor-associated macrophages of the M2 phenotype contribute to progression in gastric cancer with peritoneal dissemination. Gastric Cancer. 2016; 19:1052-65. https:// doi.org/10.1007/s10120-015-0579-8.

13. Helm O, Held-Feindt J, Schäfer H, Sebens S. M1 and M2: there is no "good" and "bad"-how macrophages promote malignancy-associated features in tumorigenesis. Oncoimmunology. 2014; 3:e946818. https://doi.org/10.416 1/21624011.2014.946818.

14. Franklin RA, Li MO. Ontogeny of tumor-associated macrophages and its implication in cancer regulation. Trends Cancer. 2016; 2:20-34. https://doi.org/10.1016/j. trecan.2015.11.004.

15. Miura T, Yoshizawa T, Hirai H, Seino H, Morohashi S, Wu Y, Wakiya T, Kimura N, Kudo D, Ishido K, Toyoki Y, Kijima H, Hakamada K. Prognostic impact of CD163+ macrophages in tumor stroma and CD8+ T-Cells in cancer cell nests in invasive extrahepatic bile duct cancer. Anticancer Res. 2017; 37:183-90. https://doi.org/10.21873/ anticanres. 11304.

16. Shi J, Li Q, Sheng M, Zheng M, Yu M, Zhang L. The role of TLR4 in M1 macrophage-induced epithelialmesenchymal transition of peritoneal mesothelial cells. Cell Physiol Biochem. 2016; 40:1538-48. https://doi. org/10.1159/000453204.
17. Ostuni R, Kratochvill F, Murray PJ, Natoli G. Macrophages and cancer: from mechanisms to therapeutic implications. Trends Immunol. 2015; 36:229-39. https://doi. org/10.1016/j.it.2015.02.004.

18. Park JY, Sung JY, Lee J, Park YK, Kim YW, Kim GY, Won KY, Lim SJ. Polarized CD163+ tumor-associated macrophages are associated with increased angiogenesis and CXCL12 expression in gastric cancer. Clin Res Hepatol Gastroenterol. 2016; 40:357-65. https://doi.org/10.1016/j. clinre.2015.09.005.

19. Karpathiou G, Casteillo F, Giroult JB, Forest F, Fournel P, Monaya A, Froudarakis M, Dumollard JM, Prades JM, Peoc'h M. Prognostic impact of immune microenvironment in laryngeal and pharyngeal squamous cell carcinoma: immune cell subtypes, immuno-suppressive pathways and clinicopathologic characteristics. Oncotarget. 2017; 8:19310-22. https://doi.org/10.18632/oncotarget.14242.

20. Noy R, Pollard JW. Tumor-associated macrophages: from mechanisms to therapy. Immunity. 2014; 41:49-61. https:// doi.org/10.1016/j.immuni.2014.06.010.

21. Bolli E, Movahedi K, Laoui D, Van Ginderachter JA. Novel insights in the regulation and function of macrophages in the tumor microenvironment. Curr Opin Oncol. 2017; 29:5561. https://doi.org/10.1097/CCO.033712R1033712R10344.

22. Tang $X$. Tumor-associated macrophages as potential diagnostic and prognostic biomarkers in breast cancer. Cancer Lett. 2013; 332:3-10. https://doi.org/10.1016/j. canlet.2013.01.024

23. Guo Q, Jin Z, Yuan Y, Liu R, Xu T, Wei H, Xu X, He S, Chen S, Shi Z, Hou W, Hua B. New mechanisms of tumorassociated macrophages on promoting tumor progression: recent research advances and potential targets for tumor immunotherapy. J Immunol Res. 2016; 2016:9720912. https://doi.org/10.1155/2016/9720912.

24. Ries CH, Cannarile MA, Hoves S, Benz J, Wartha K, Runza V, Rey-Giraud F, Pradel LP, Feuerhake F, Klaman I, Jones T, Jucknischke U, Scheiblich S, et al. Targeting tumorassociated macrophages with anti-CSF-1R antibody reveals a strategy for cancer therapy. Cancer Cell. 2014; 25:846-59. https://doi.org/10.1016/j.ccr.2014.05.016.

25. Mahmoud SM, Lee AH, Paish EC, Macmillan RD, Ellis IO, Green AR. Tumour-infiltrating macrophages and clinical outcome in breast cancer. J Clin Pathol. 2012; 65:159-63. https://doi.org/10.1136/jclinpath-2011-200355.

26. Liu JY, Yang XJ, Geng XF, Huang CQ, Yu Y, Li Y. Prognostic significance of tumor-associated macrophages density in gastric cancer: a systemic review and metaanalysis. Minerva Med. 2016; 107:314-21.

27. Marech I, Ammendola M, Sacco R, Sammarco G, Zuccalà V, Zizzo N, Leporini C, Luposella M, Patruno R, Filippelli G, Russo E, Porcelli M, Gadaleta CD, et al. Tumourassociated macrophages correlate with microvascular bed extension in colorectal cancer patients. J Cell Mol Med. 2016; 20:1373-80. https://doi.org/10.1111/jcmm.12826. 
28. Hu WQ, Fang M, Zhao HL, Yan SG, Yuan JP, Peng CW, Yang GF, Li Y, Li JD. Tumor invasion unit in gastric cancer revealed by QDs-based in situ molecular imaging and multispectral analysis. Biomaterials. 2014; 35:4125-32. https://doi.org/10.1016/j.biomaterials.2014.01.059.

29. Su S, Liu Q, Chen J, Chen J, Chen F, He C, Huang D, Wu W, Lin L, Huang W, Zhang J, Cui X, Zheng F, et al. A positive feedback loop between mesenchymal-like cancer cells and macrophages is essential to breast cancer metastasis. Cancer Cell. 2014; 25:605-20. https://doi. org/10.1016/j.ccr.2014.03.021.

30. Llosa NJ, Cruise M, Tam A, Wicks EC, Hechenbleikner EM, Taube JM, Blosser RL, Fan H, Wang H, Luber BS, Zhang M, Papadopoulos N, Kinzler KW, et al. The vigorous immune microenvironment of microsatellite instable colon cancer is balanced by multiple counter-inhibitory checkpoints. Cancer Discov. 2015; 5:43-51. https://doi. org/10.1158/2159-8290.CD-14-0863.

31. Roxburgh CS, McMillan DC. The role of the in situ local inflammatory response in predicting recurrence and survival in patients with primary operable colorectal cancer. Cancer Treat Rev. 2012; 38:451-66. https://doi.org/10.1016/j. ctrv.2011.09.001.

32. Murray PJ, Allen JE, Biswas SK, Fisher EA, Gilroy DW, Goerdt S, Gordon S, Hamilton JA, Ivashkiv LB, Lawrence T, Locati M, Mantovani A, Martinez FO, et al. Macrophage activation and polarization: nomenclature and experimental guidelines. Immunity. 2014; 41:14-20. https:// doi.org/10.1016/j.immuni.2014.06.008.

33. Italiani $\mathrm{P}$, Boraschi D. From monocytes to $\mathrm{M} 1 / \mathrm{M} 2$ macrophages: phenotypical vs. functional differentiation. Front Immunol. 2014; 5:514. https://doi.org/10.3389/ fimmu.2014.00514.

34. Komohara Y, Jinushi M, Takeya M. Clinical significance of macrophage heterogeneity in human malignant tumors. Cancer Sci. 2014; 105:1-8. https://doi.org/10.1111/ cas. 12314
35. Lim SY, Yuzhalin AE, Gordon-Weeks AN, Muschel RJ. Tumor-infiltrating monocytes/macrophages promote tumor invasion and migration by upregulating S100A8 and S100A9 expression in cancer cells. Oncogene. 2016; 35:5735-45. https://doi.org/10.1038/onc.2016.107.

36. Zhou D, Huang C, Lin Z, Zhan S, Kong L, Fang C, Li J. Macrophage polarization and function with emphasis on the evolving roles of coordinated regulation of cellular signaling pathways. Cell Signal. 2014; 26:192-7. https:// doi.org/10.1016/j.cellsig.2013.11.004.

37. Qian BZ, Pollard JW. Macrophage diversity enhances tumor progression and metastasis. Cell. 2010; 141:39-51. https:// doi.org/10.1016/j.cell.2010.03.014.

38. Pinto ML, Rios E, Silva AC, Neves SC, Caires HR, Pinto AT, Durães C, Carvalho FA, Cardoso AP, Santos NC, Barrias CC, Nascimento DS, Pinto-do-Ó P, et al. Decellularized human colorectal cancer matrices polarize macrophages towards an anti-inflammatory phenotype promoting cancer cell invasion via CCL18. Biomaterials. 2017; 124:211-24. https://doi.org/10.1016/j.biomaterials.2017.02.004.

39. Tiainen S, Tumelius R, Rilla K, Hämäläinen K, Tammi M, Tammi R, Kosma VM, Oikari S, Auvinen P. High numbers of macrophages, especially M2-like (CD163-positive), correlate with hyaluronan accumulation and poor outcome in breast cancer. Histopathology. 2015; 66:873-83. https:// doi.org/10.1111/his.12607.

40. Chen JM, Qu AP, Wang LW, Yuan JP, Yang F, Xiang QM, Maskey N, Yang GF, Liu J, Li Y. New breast cancer prognostic factors identified by computer-aided image analysis of HE stained histopathology images. Sci Rep. 2015; 5:10690. https://doi.org/10.1038/srep10690.

41. Colegio OR, Chu NQ, Szabo AL, Chu T, Rhebergen AM, Jairam V, Cyrus N, Brokowski CE, Eisenbarth SC, Phillips GM, Cline GW, Phillips AJ, Medzhitov R. Functional polarization of tumour-associated macrophages by tumourderived lactic acid. Nature. 2014; 513:559-63. https://doi. org/10.1038/nature13490. 